Dr. Francisco López-Cantos

Universitat Jaume I

@ flopez@uji.es
Recibido / Received 20 de septiembre de 2018

- Aceptado / Acepted 6 de noviembre de 2018

- Páginas / Pages De la 39 a la 53

—ISSN: 1885-365X

\title{
Del rigor a la retórica emocional. Comunicación y discurso educativo TEDTalk para el aula
}

From rigor to emotional rhetoric. Communication and educational TEDTalk discourse for the classroom.

En el presente trabajo se analizan las estrategias discursivas que se utilizan en las TEDTalks utilizando el análisis de contenido como metodología de investigación. Nuestro análisis concluye que los contenidos de las TEDTalks educativas tienen escasa utilidad real para su aplicación en las aulas más allá de aportar algunas anotaciones, por otro lado ya conocidas por los docentes y presentes entre las propuestas de enseñanza-aprendizaje contemporáneas, y mejor evitar la panacea del solucionismo simple a cuestiones educativas complejas y la particular ideología MIT que se promueve en este tipo de discursos.

PALABRAS CLAVE: TED, estrategias de enseñanza, análisis del discurso, innovación educativa, competencias comunicativas, empoderamiento, plataforma digital, cultura digital.

This paper analyzes TEDTalks discursive strategies using content analysis as a research methodology. Our analysis concludes that the contents of the educational TEDTalks have little real usefulness for their application in classrooms beyond providing some annotations already known by teachers and present among contemporary teaching-learning proposals, and better avoid the panacea of simple solutionism to complex educational issues and the particular MIT ideology that is promoted in this type of discourses.

KEY WORDS: teaching strategies, discourse analysis, educational innovation, communication skills, empowerment, digital platform, digital culture.

\section{Introducción y estado de la cuestión}

Los nuevos escenarios educativos están insertos, obviamente, en procesos socioculturales más amplios y actualmente altamente tecnificados que imponen la renovación sistémica en multitud de áreas del conocimiento, y no sólo en lo relativo a los sistemas didácticos y las estrategias pedagógicas tradicionales.

En este sentido, y como consecuencia del desarrollo de la Web 2.0, la nueva sociedadred actual se ha definido, primero, como "sociedad del conocimiento" (Gibbons et al., 1994), 
en la que la ciencia y la tecnología producen profundos cambios en las relaciones sociales y económicas (Knorr-Cetina, 1981) para, después, evidenciar los cambios que se están produciendo para la conformación de las actuales "economías basadas en el conocimiento" construidas a partir de "redes epistémicas" (Ibarra Unzueta, 2012). Y la renovación de los procesos de enseñanza-aprendizaje es, obviamente, imprescindible para adecuar los actuales entornos educativos a los profundos cambios que se están produciendo en esta compleja dinámica sociocultural contemporánea.

Las nuevas estrategias pedagógicas hacen uso, como no puede ser de otra manera, de las posibilidades que permite la tecnología multimedia y las redes de comunicación y en el ámbito educativo se impulsan cada vez con mayor éxito las propuestas asociadas al fomento del aprendizaje activo y colaborativo. De este modo, se están implementando estrategias y herramientas pedagógicas enquiry-based (Wang, 2012) y gaming-based learning (Albretch, 2012), así como innovadoras técnicas que fomentan la cooperación y la implicación de los estudiantes como las integradas en la metodología de enseñanzaaprendizaje denominada flipped-classroom (Baker, 2000; Bergmann y Sams, 2014; Strayer, 2014; Abeysekera y Dawson, 2015; Tourón y Santiago, 2015). Aunque todavía existe cierta controversia acerca de la eficacia real de estas nuevas estrategias pedagógicas respecto a las metodologías clásicas (Boeve et al., 2017) o en relación con el uso de los nuevos entornos online para el aprendizaje masivo denominados MOOC (Bartolomé y Steffens, 2015), parece ser que cuanto menos las metodologías didácticas mas vanguardistas impulsan la participación activa y colaborativa de los estudiantes y, por extensión, facilitan mejoras en los resultados de aprendizaje. Incluso en algunos entornos educativos se están adoptando técnicas más propias de la gestión empresarial como el coaching (Bandura, 1997; Harvard Business Esentials, 2005; Wolk, 2007; Giraldez Hayes y Van Nieuwerburgh, 2016) orientadas, en igual sentido, a potenciar el desarrollo y la capacidad de aprendizaje autónomo de los estudiantes.

Las nuevas formas de entender la enseñanza adquieren últimamente además diversas denominaciones en las que, como ocurre en otras áreas de conocimiento, predominan las que contienen el prefijo de moda neuro-, y que suponen la progresiva asimilación y aplicación, con mayor o menor fortuna (y oportunismo), del conocimiento acumulado de la investigación en ciencias cognitivas a lo largo de las últimas décadas. En lo que nos compete, al ser la enseñanza-aprendizaje un proceso de carácter cognitivo-relacional entendemos que resulta pertinente utilizar estrategias educativas fundamentadas en redes de interacción colaborativa que sean multimodales y multimediales. Tal como se concluye en un reciente estudio, es necesario contemplar el papel central que tienen las emociones y la comunicación persuasiva en la comunicación educativa. Es decir, en los procesos de enseñanza-aprendizaje es fundamental tener en cuenta la dimensión emocional y potenciar el relato, y la habilidad más preciada de un educador comunicativo es su «capacidad de movilización, implicación e interacción» (Ferres y Masanet, 2017, p. 60).

La actual renovación pedagógica, como no puede ser de otra manera y tal como ocurre en todos los ámbitos, está impulsada y condicionada por tecnologías audiovisuales y online cuya inevitable aplicación durante los procesos sistémicos de transformación siempre genera no pocas controversias y su inclusión en los actuales modelos educativos acaba siendo el resultado de décadas de una lenta e inevitable transformación sistémica. Como hacía notar 
Bruno Latour (2009, p. 155): «Change the instruments, and you will change the entire social theory that goes with them».

Una de las más interesantes muestras de la profunda transformación educativa que se impulsa con la aplicación de renovadas estrategias comunicativas y nuevas herramientas tecnológicas lo representan las conocidas TEDTalks, un inédito género de comunicación educativa que está provocando no pocas controversias y parece estar revolucionando las tradicionales formas de enseñanza-aprendizaje haciendo uso de plataformas online para difusión de contenidos audiovisuales de carácter científico/educativo.

No está entre los objetivos de este breve trabajo dirimir la complejidad de aspectos inherentes a la comunicación pública del conocimiento, al respecto se puede consultar abundante literatura y multitud de textos específicos sobre los cambios contemporáneos en las formas de difusión de la ciencia (Trench, 2007; Schäfer, 2009; Suleski y lbaraki, 2010; Bennett y Jennings, 2011; Moreno-Castro, 2011; López-Pérez y Olvera-Lobo, 2016; LópezCantos, 2017) pero, en cualquier caso, es indudable la utilidad de los contenidos audiovisuales para la enseñanza tal como se muestra en numerosos trabajos desde hace décadas (Bravo Ramos, 1996; Bartolomé, 2003), y su eficacia didáctica en el actual entorno online (MeseguerMartínez, Ros-Gálvez y Rosa-García, 2017) y para la divulgación científica (Allgaier, 2013), así como la demostrada capacidad didáctica en diversos ámbitos educativos que tiene la plataforma Youtube (Desmet, 2009; Jones y Cuthrell, 2011; Jaffar, 2012; Kulgemeyer y Peters, 2016) o las posibilidades que ofrece el video online para desarrollar novedosas estrategias docentes utilizando herramientas colaborativas (McClean et al., 2016).

Tal como TED se define a así mismo:

TED is a nonprofit devoted to spreading ideas, usually in the form of short, powerful talks (18 minutes or less). TED began in 1984 as a conference where Technology, Entertainment and Design converged, and today covers almost all topics -from science to business to global issues - in more than 100 languages. Meanwhile, independently run TEDx events help share ideas in communities around the world (Ted.com, 2017)

Y con esta filosofía que ha variado poco desde las primeras conferencias que se celebraban en Monterrey (California) a la eclosión que se produjo en 2006 con el lanzamiento de la plataforma online, el fenómeno TED se ha extendido globalmente y no deja de crecer. En la actualidad se ha constituido en un conglomerado de productos que comprende desde las propias TEDTalks que se difunden online a través de la plataforma propia y en el canal de Youtube, a otros destinados al entorno educativo como el recientemente lanzado TED-Ed, y otras conferencias similares franquiciadas localmente con la denominación TEDx.

Desde su relanzamiento, TED es propiedad de una organización sin ánimo de lucro, Sapling Foundation, pero en la que, como han criticado sus detractores, sus ejecutivos tienen salarios anuales que superan las siete cifras (Segar, 2011). El privilegio de poder ser seleccionado para asistir como público a una conferencia TEDTalk entre las programadas para el año 2018 tiene un coste económico que va desde los 5.000 hasta los 250.000 dólares que aportan los patronos TED y con los que obtienen ventajas promocionales adicionales. Las conferencias TEDTalks que son seleccionadas por la organización para su inclusión en la plataforma online se distribuyen junto con su transcripción textual a numerosas lenguas y en un blog en que se promueven los comentarios de los usuarios, facilitando la interacción 
directa de los usuarios y la creación de una comunidad discursiva online formada por los millones de seguidores de TED en todo el mundo.

El interés académico en el fenómeno TED ha ido aumentando al tiempo que la organización iba creciendo y teniendo cada vez un impacto más global, y en fechas recientes se ha analizado su eficacia tanto como herramienta educativa (DaVia Rubenstein, 2012) como para la popularización de la ciencia (Sugimoto y Thelwall, 2013; Welbourne y Grant, 2016) y potencialmente transformadora, aunque solo en apariencia como demuestran sus críticos (Denskus y Esser, 2015). En este sentido, y en un entorno de profundos cambios en cuanto a la percepción de la validez social del conocimiento científico (Buchhi, 2017; Zorn et al., 2012), las limitaciones de estas nuevas formas de popularización del conocimiento están siendo cada vez mas puestas en evidencia (Kaeser, 2013; Scharrer et al., 2016). Y, aunque de un lado se publican manuales que difunden la estrategia comunicativa y promueven la supuesta eficacia del modelo TED y la labor de la plataforma en la difusión del conocimiento (Donovan, 2014), son numerosas también las críticas al formato y sus contenidos, a veces en forma de parodias (Feirstein, 2017), por considerarse poco más que un mero producto de marketing que promueve la "locura del solucionismo tecnológico" propia de la "ideologia MIT" (Bratton, 2013; (Morozov, 2013).

Por otra parte y más allá de estas controversias, algunos investigadores, defienden que las TEDTalk han llegado a constituirse en sí mismas en un nuevo y genuino género comunicativo/educativo híbrido resultante de la combinación de la tradicional conferencia académica con la presentación comercial y los relatos de memorias y biográficos (Ludewig, 2017). Las TEDTalks se caracterizan por su rígida planificación y estructuración previa y por su limitación temporal (alrededor de 18 minutos aconsejan en la organización, aunque esta indicación se cumple poco), así como por potenciar los resultados más que los métodos y por la utilización de elementos discursivos que promueven la proximidad con el público como estrategia persuasiva para la comunicación de la ciencia (Scotto di Carlo, 2014a). En igual sentido, algunos trabajos han analizado las características formales de las conferencias TEDTalk (Kedrowicz y Taylor, 2016) y su impacto en las audiencias (Tsou et al., 2014), así como también las diferencias prosódicas en la exposición oral que determinan el éxito de los conferenciantes TED frente a los profesores universitarios (Tsai, 2015) o el uso que se hace del humor (Scotto di Carlo, 2014b) y la utilización de contenidos metafóricos (Mijin, 2014).

En el momento de redacción de este artículo se recopilan más de 2.600 TEDTalks seleccionadas específicamente para la plataforma y accesibles online entre todas las que se han realizado a lo largo de los años. Las mas populares superan los 15-20 millones de visitas y se alcanzan cifras acumuladas cercanas al billón y medio de visitas totales. La mas difundida de todos los tiempos, con más de 48 millones de visitas hasta el momento, está dedicada a la educación y pretende responder a la pregunta "Do schools kill creativity?" (Robinson, 2006), y entre las primeras que se difundieron se encuentra la visionara en que Nicholas Negroponte, fundador del MIT, predecía el futuro de la tecnología (Negroponte, 1984) o, en la que años después Al Gore alertaba sobre el cambio climático (AI Gore, 2006), todas sin duda anticipatorias de los debates contemporáneos en los que estamos inmersos y correspondientes con el propio espiritu fundacional que define las charlas TED: "Ideas worht spreading".

En esta investigación, vamos a analizar las estrategias discursivas que se utilizan en las TEDTalks y que facilitan que tengan tanto éxito para, de un lado, identificar los elementos 
comunicativos que pueden ser de interés general para la comunicación pública del conocimiento y, por extensión, determinar la oportunidad de adoptar estrategias similares para mejorar el proceso de enseñanza-aprendizaje en el aula.

La hipótesis principal ( $\mathrm{H} 1)$ de esta investigación es que las estrategias comunicativas de proximidad con la audiencia determinan la eficacia de las TEDTalk y que estos recursos podrían ser de interés para mejorar la docencia en el aula. De manera complementaria, y como segunda hipótesis ( $\mathrm{H} 2)$, queremos mostrar que el modelo de enseñanza-aprendizaje que se promueve en las TEDTalk es el resultado de un específico marco conceptual que como, ya hemos mencionado, se viene denominando "ideología MIT".

\section{Material y métodos}

Para nuestro estudio vamos a analizar la lista de reproducción de TEDTalks que la propia organización ha elaborado con el título Talks from inspiring teaching (https://www.ted.com/ playlists/182/talks_from_inspiring_teachers), y que se presenta como «A great teacher can change the trajectory of your life. Here, talks from teachers you'll wish you had». La muestra de análisis tiene una duración total de 1h:49m:34s y está formada por las diez conferencias que han sido seleccionadas por la propia organización como inspiradoras y que han sido reproducidas alrededor de 22 millones de veces en total y, por lo tanto, son representativas del modelo educativo que se promueve desde la plataforma TED y de su impacto global.

Sobre la muestra de estudio realizamos, de una parte, un análisis de contenido a partir de las transcripciones textuales de las conferencias para determinar los marcos interpretativos que propone y las estrategias discursivas que utiliza. En segundo lugar, y de manera complementaria, realizamos un análisis formal del dispositivo audiovisual con el que se distribuyen las TEDTalk en la plataforma online para determinar la estrategia discursiva que utilizan. Partiendo de los postulados teóricos propios de la Frame Theory (Goffman, 1974), con la perspectiva metodológica down-up que propone la Grounded Theory (Glasser y Strauss, 1967), y con la ayuda del software de codificación y análisis de contenido Atlas. ti, determinamos el contenido discursivo que promueven las TEDTalk seleccionadas para identificar los elementos que facilitan su eficacia comunicativa y su enorme éxito.

\section{Análisis y resultados}

El análisis se ha realizado sobre un total de 15.599 partículas lingüísticas a partir de la transcripción en inglés del conjunto de TEDTalks que, en primer lugar y en términos cuantitativos, han dado como resultado, una vez depuradas las partículas no significativas, una clara predominancia de elementos que son propios de estrategias discursivas de proximidad, y se observan continuas interpelaciones a la audiencia y al nosotros y, también, una clara personalización en el propio ponente y una evidente objetualización de la otredad ajena a la comunidad TED. 


\begin{tabular}{|l|l|l|}
\hline Word & Count & Percent \\
\hline you & 283 & $2 \%$ \\
\hline we & 252 & $1 \%$ \\
\hline my & 195 & $1 \%$ \\
\hline they & 172 & $1 \%$ \\
\hline
\end{tabular}

De este modo, son numerosos los ejemplos en que se busca establecer un vínculo de proximidad con la audiencia proponiendo específicos marcos cognitivos que faciliten la conformación de una comunidad discursiva diferencial y propia, en muchos casos para la identificación de problemas y propuesta de soluciones y, siempre, como medio para promover con insistencia en el empoderamiento de la audiencia, por ejemplo:

«If our children are not in our classrooms, how will they learn? And if they're not learning, where would they end up?» (Why an open school? To close a prison, 01:33).

«Is this job tough? You betcha. Oh God, you betcha. But it is not impossible. We can do this. We're educators. We're born to make a difference» (Every kid needs a champion, 07:24).

«But I'm here to tell you that magic can be taught. Magic can be taught. Magic can be taught. Now, how do you teach it? You teach it by allowing people to go into those spaces where the magic is happening. If you want to be an aspiring teacher in urban education, you've got to leave the confines of that university and go into the hood" (Teach teachers how to create magic, 05:24).

En este último ejemplo resulta significativa la interpelación de carácter religioso que se hace a las audiencias: «Can I get an Amen?» (Teach teachers how to create magic, 4:18) como muestra de las importantes diferencias de los marcos discursivos TEDTalks en relación con nuestro propio entorno cultural.

En cuanto a la estructura discursiva de las charlas esta no es del todo idéntica en todas ellas, pero sí se distingue con claridad una estrategia argumentativa orientada a resultados con un modelo identificación/solución problema/resultados obtenidos y que acaba siempre promoviendo la comunidad discursiva y el empoderamiento. Y en todos los casos, y en línea con la búsqueda de cercanía con la audiencia, las charlas se inician contando anécdotas o experiencias personales, algunas veces acompañadas de efectos para captar la atención, que sirven para contextualizar e identificar el problema al que se va a dar posterior solución, por ejemplo:

«Let me tell you a story».

«It's my first year as a new high school science teacher, and I'm so eager. I'm so excited, I'm pouring myself into my lesson plans. But I'm slowly coming to this horrifying realization that my students just might not be learning anything». (Hey science teachers - make it fun, 00:16). 
«I teach chemistry. (Explosion). All right, all right. So more than just explosions, chemistry is everywhere. Have you ever found yourself at a restaurant spacing out just doing this over and over? Some people nodding yes.Recently, I showed this to my students, and I just asked them to try and explain why it happened. The questions and conversations that followed were fascinating» (3 rules to spark learning, 00:12).

«My mother had 16 pregnancies. From 16 pregnancies, five of us are alive. You can imagine as a child what I went through. Day to day, I watched women being carried to a graveyard, or watched children going to a graveyard" (How I stopped the Taliban from shutting down my school, 00:42).

Durante el transcurso de cada charla se recurre de manera habitual a la cita de autoridad, a Mark Twain, y Martin Luther King por ejemplo entre otros, buscando así consolidar la solvencia del ponente, pero el efecto de credibilidad se consigue sobre todo a partir del relato de experiencias o anécdotas personales.

Es interesante señalar también la clara orientación a resultados que se muestra en la mayoría de las charlas como efecto de este solucionismo con que se estructura argumentativamente el dispositivo discursivo, por ejemplo:

«The results? After one year, state data revealed that our scores have grown by 171 percent in Algebra and 107 percent in literature. (Applause) We have a very long way to go, a very long way to go, but we now approach every obstacle with a "So what. Now what?" attitude» (How to fix a broken school, 10:18).

De nuestro análisis se desprende igualmente y de manera muy evidente el significativo uso que se hace del humor como estrategia persuasiva que sirve como elemento dramático a partir del cual se despliega de manera progresiva toda la estructura narrativa hasta llegar al climax final del empoderamiento. En muchas ocasiones las risas de la audiencia son acompañadas por aplausos en una suerte de exitoso momentum en que cristaliza la buscada conformación de una comunidad discursiva única y original en torno a la charla TED que se está poniendo en acto, aunque como podemos observar en los datos el recurso al humor facilita el éxito, no es garantía absoluta del interés de la charla en la audiencia:

\begin{tabular}{|l|l|l|l|}
\hline TEDTalk & Laughter & Applause & Visionados \\
\hline $\begin{array}{l}\text { Every kid needs a champion } \\
\text { How to fix a broken school? Lead fearlessly, }\end{array}$ & 9 & 3 & $7,738,931$ \\
\hline $\begin{array}{l}\text { love hard } \\
\text { How I stopped the Taliban from shutting down }\end{array}$ & 8 & 8 & $1,676,912$ \\
\hline $\begin{array}{l}\text { my school } \\
\text { Math class needs a makeover }\end{array}$ & 4 & 7 & $1,387,567$ \\
\hline
\end{tabular}




\begin{tabular}{|l|l|l|l|}
\hline Why open a school? To close a prison & 1 & 3 & $1,057,005$ \\
\hline How to learn? From mistakes & 1 & 1 & $1,926,957$ \\
\hline A teacher growing green in the South Bronx & 1 & 1 & $1,142,879$ \\
\hline rules to spark learning & 0 & 1 & $2,678,251$ \\
\hline Hey science teachers - make it fun & 0 & 1 & $1,530,872$ \\
\hline Teach teachers how to create magic & 0 & 1 & $2,049,947$ \\
\hline
\end{tabular}

En este sentido, a lo largo de cada una de las charlas y siguiendo esta estructura argumentativa que decimos de manera más o menos lineal, es mucho más efectivo el que se vayan sucediendo anécdotas y confesiones personales con la misma función discursiva doble, es decir, buscar cercanía de la audiencia y contextualizar los problemas a solventar hasta concluir con su resolución de manera sintética dando indicaciones y empoderando a la audiencia y a veces con el recurso a sloganes más o menos efectivos y populares, por ejemplo:

\begin{abstract}
«And as we lead, we must never forget that every single one of our students is just a child, often scared by what the world tells them they should be, and no matter what the rest of the world tells them they should be, we should always provide them with hope, our undivided attention, unwavering belief in their potential, consistent expectations, and we must tell them often, if nobody told them they loved them today, remember we do, and we always will» (How to fix a broken school, 15:42).
\end{abstract}

«When tenacious little ones learn how to garden like this, it's no wonder we get fruit like that. And I love it! And so do they. And we're building teepees in neighborhoods that were burning down. And that's a true "sí se puede" moment» (A teacher growing green in the South Bronx, 08:15).

De esta manera, y en conjunto, los resultados de nuestro análisis muestran una clara organización discursiva y una minuciosa puesta en escena en cada una de las charlas que acaba en el climax de una cerrada ovación ritual en que se pasa a formar parte de la comunidad resolutiva TED. El éxito se consigue con el recurso al efecto de cercanía a partir de una estrategia argumentativa orientada a ofrecer soluciones y resultados en el último tramo del dispositivo discursivo para, finalmente, acabar convenciendo a la audiencia de su potencial transformador global.

Las soluciones que se ofrecen a problemas complejos, tal como se puede observar con claridad, resultan ser mas bien un catálogo de proposiciones voluntariosas a las que es difícil que la audiencia no se adscriba:

\footnotetext{
«Improve and build better relationships»" (Every kid needs a champion).

«Better preparation of teaching on real places» (Teach teachers how to create magic).

«Lead, manage problems and love students» (How to fix a broken school).
} 
«Telling histories» (Hey science teachers - make it fun).

"Involve students to be useful and practical learning and use multimedia resources" (Math class needs a makeover).

«Embrace a new paradigm as cultivators of curiosity and inquiry, we just might bring a little bit more meaning to their school day, and spark their imagination» (3 rules to spark learning).

«Training teacher and complete their potential, having compassion and having love» (How I stopped the Taliban from shutting down my school).

"Go from the audacity to hope to hope for some audacity, use your energy, teach entrepeneurship" (A teacher growing green in the South Bronx).

«Provide love, structure, support and knowledge to inspire children» (Why open a school? To close a prison).

«Promote experiential learning, empowering student voice and embracing failure» (How to learn? From mistakes).

Y, resultado de todo ello y como se puede observar en nuestro análisis, con grandes dosis de simpleza y esloganes a los que fácilmente la audiencia se puede adherir y conformar ese momentum comunal no resulta nada complicado para conseguir la ovación ritual final buscada tras la calculada progresión dramática con que se desarrolla el discurso y, por extensión, el éxito de la charla está asegurado.

\section{Discusión y conclusiones}

Tal como se desprende de nuestro análisis podemos concluir, en primer lugar, que nuestra hipótesis de partida $(\mathrm{H} 1)$ es correcta y los resultados muestran que la estrategia persuasiva que define el modelo comunicativa propio de las TEDTalks esta determinada por el uso de recursos discursivos cuya función es promover la cercanía con la audiencia y con ello se facilita la conformación de una comunidad discursiva propia que asegura el éxito de la plataforma TED y promueve con efectividad su difusión global.

En esta estrategia persuasiva, juega un papel determinante tanto el uso del humor, en consonancia con lo que han mostrado otros trabajos al respecto (Scotto di Carlo, 2014b), como, sobre todo, la utilización recursos narrativos dramáticos en forma de anécdotas o experiencias personales del ponente que se despliegan de manera estructurada a lo largo de discurso para concluir con eslóganes simples que buscan el empoderamiento colectivo y la adhesión emocional de la audiencia.

A pesar del éxito de audiencia que tiene esta influyente plataforma comunicativa global, el modelo transformador educativo TEDTalk tiene capacidad social transformadora nula, como otros autores han mostrado (Denskus y Esser, 2015), ni ofrece en sí mismo un modelo educativo como tal si no únicamente experiencias sesgadas y parciales que no solo no abordan la complejidad del proceso educativo si no que, además, se pretenden novedosos y brillantes cuando no son más que meras simplificaciones de bien conocidas estrategias de enseñanza-aprendizaje que se vienen implementando en nuestras aulas.

En cuanto a la segunda hipótesis que queríamos contrastar (H2), hemos mostrado que las 
TEDTalks sobre educación analizadas presentan características que son más propias de los discursos motivacionales que de los académicos y no son más que un cúmulo de eslóganes motivacionales simples. En ese sentido nuestros resultados son concurrentes con las críticas al universo discursivo TED por estar fundamentando en el solucionismo tecnológico propio de la "ideología MIT" (Bratton, 2013; Morozov, 2013).

Entendemos, finalmente y como conclusión de nuestro análisis, que los contenidos de las TEDTalks analizadas tienen escasa utilidad real para su aplicación en las aulas más allá de aportar algunas anotaciones, por otro lado ya conocidas por los docentes y presentes entre las propuestas metodológicas de enseñanza-aprendizaje contemporáneas, acerca de la forma más efectiva de utilizar los recursos comunicativos propios del discurso argumentativo/ persuasivo y las estrategias de proximidad en conjunción con herramientas multimedia.

Es obvio que los sistemas educativos pueden y deben mejorar y es necesario seguir aportando soluciones y renovando las metodologías docentes que, sin duda, pasan por potenciar la implicación de los alumnos con la ayuda de tecnologías colaborativas y online y, en general, mejorar las estrategias comunicativas en el aula (Ferres y Masanet, 2017). Pero la panacea de solucionismo simple a cuestiones educativas complejas, y a los problemas contemporáneos en general, que ofrecen las TEDTalk analizadas y su capacidad revolucionaria no es, a nuestro entender, más que el preocupante síntoma de la expansión global de un particular mitología discursiva que al parecer nos salvará de todo y gira en torno a la creatividad e innovación transformadora (Pfothenhauer y Jasanoff, 2017), y que no hace más que confirmar un diagnostico poco alentador acerca de las estructuras sobre las que se expande y consolida el poder políticoeconómico global (Foucault, 1966; Lyotard, 1979; Mattelard, 1996) en nuestra contemporaneidad.

\section{Apoyos}

Esta investigación no ha recibido ayudas públicas o privadas.

\section{Bibliografía}

ABEYSEKERA, Lakmal y DAWSON, Phillip (2015). Motivation and cognitive load in the flipped classroom: definition, rationale and a call for research. Higher Education Research y Development, 34(1), 1-14. DOI: 10.1080/07294360.2014.934336

ALBRETCH, Sophie Charlotte (2012). The game of happiness. Gamification of positive activity interventions. Maastricht: Maastricht University.

ALLGAIER, Joachim (2013). On the shoulders of YouTube. Science Communication, 35(2), 266-75. DOI: $10.1177 / 1075547012454949$

BAKER, J. Wesley (2000). The "Classroom Flip": Using web course management tools to become the guide by the side. Proceedings of 11th International conference on college teaching and learning, 9-17. Recuperado de: http://www.classroomflip.com/files/classroom_flip_baker_2000.pdf 
BANDURA, Albert (1997). Self-efficacy: The exercise of control. New York: W.H. Freeman.

BARTOLOMÉ, Antonio (2003). Vídeo digital. Comunicar, XI(21), 39-47. Recuperado de: https://www. revistacomunicar.com/verpdf.php?numero $=21$ yarticulo $=21-2003-06$

BARTOLOMÉ, Antonio y STEFFENS, Köln (2015). ¿Son los MOOC una alternativa al aprendizaje. Comunicar, XXII(44), 91-99. DOI: 10.3916/C44-2015-10

BENNET, David y JENNINGS, Richard (Eds.) (2011). Succesful science communication. Cambridge: Cambridge University Press.

BERGMANN, Jonathan y SAMS, Aaron (2012). Flip your classroom: Reach every student in every class every day. Washington: ISTE.

BOEVÉ, Anja, MEIJER, Rob, BOSKER, Roel, VUGTEVEEN, Jorien, HOEKSTRA, Rind y ALBERS, Casper J. (2017). Implementing the flipped classroom: and exploration of study behaviour and student performance. Higher Education, 74 (6), 1015-1032. DOI: 10.1007/s10734-016-0104-y

BRATTON, Benjamin (2013). We need to talk about TED. The Guardian. Recuperado de: http://www. theguardian.com/commentisfree/2013/dec/30/we-need-to-talk-about-ted

BRAVO RAMOS, Luis (1996). ¿Qué es el vídeo educativo?. Comunicar, IV(6), 100-106. Recuperado de: https://www.revistacomunicar.com/verpdf.php?numero=6yarticulo=06-1996-20

BUCCHI, Massimiano (2017). Credibility, expertise and the challenges of Science Communication 2.0. Public Understanding of Science, 26(8), 890-93. DOI: 10.1177/0963662517733368

DAVIA RUBENSTEIN, Lisa (2012). Using TED Talks to Inspire Thoughtful Practice. Teacher Educator, 47(4), 261-67. DOI: 10.1080/08878730.2012.713303

DENSKUS, Tobias y ESSER, Daniel (2015). TED Talks on international development: Trans-Hegemonic promise and ritualistic constraints. Communication Theory, 25(2), 166-87. DOI: 10.1111/comt.12066

DESMET, Christy (2009). Teaching Shakespeare with YouTube. English Journal, 99(1), 65-70. Recuperado de: http://www.csun.edu/ krowlands/Content/Academic_Resources/Technology/ Desmet-Teaching\%20Shakespeare\%20with\%20YouTube.pdf

DONOVAN, Jeremey (2014). How to deliver a TED talk: Secrets of the world's most inspiring presentations. New York: McGraw Hill Education.

FERRES, Joan y MASANET, María-José (2017). La eficacia comunicativa en la educación: potenciando las emociones y el relato. Comunicar, XXV(52), 51-60. DOI: 10.3916/C52-2017-05

FEIRSTEIN, Bruce (2013). The Ted-O-Matic! How to generate your own, faux-profound TED Talk. Vanity Fair. Recuperado de: https://www.vanityfair.com/news/2013/04/ted-conference-talks-humor 
FOUCAULT, Michel (1966). Les mots et les choses. Paris: Ed. Gallimard.

GOFFMAN, Erving (1974). Frame analysis: An essay on the organization of experience. New York: Harper y Row.

GIBBONS, Michael, LIMOGES, Camille, NOWOTNY, Helga, SCOT, Peter y TROW, Martin (1994). The new production of knowledge: The dynamics of science and research in contemporary societies. London: SAGE.

GIRALDEZ HAYES, Andrea y VAN NIEUWERBURGH, Christian (2016). Coaching educativo. Madrid: Paraninfo.

GLASSER, Barney y STRAUSS, Anselm (1967). The discovery of grounded theory strategies for qualitative research. Chicago: Edit. Aldine Pub.

GORE, Al (2006). Averting the climate crisis. TED Ideas Worth Spreading. Recuperado de: https://www.ted. com/talks/al_gore_on_averting_climate_crisis

Harvard Business Essentials (2005). Coaching y mentoring: cómo desarrollar el talento de alto nivel y conseguir mejores resultados. Barcelona: Ediciones Deusto.

IBARRA UNZUETA, Andoni (2012). Epistemic networks. New Subjects for new forms of (scientific) knowledge production. Science, Technology and Innovation Studies, 8(1), 61-74.

JAFFAR, Akram Abood (2012). YouTube: An emerging tool in anatomy education. Anatomical Sciences. Education, 5(3), 158-164. DOI: 10.1002/ase.1268

JONES, Troy y CUTHRELL, Kristen (2011). YouTube: Educational potentials and pitfalls. Computers in the schools, 28(1), 75-85. DOI: 10.1080/07380569.2011.553149

KAESER, Eduard (2013). Science kitsch and pop science: A reconnaissance. Public Understanding of Science, 22(5), 559-569. DOI: 10.1177/0963662513489390

KEDROWICZ, April y TAYLOR, Julie (2016). Shifting rhetorical norms and electronic eloquence: TED talks as formal presentations. Journal of Business and Technical Communicationm, 30(3), 352-377. DOI: $10.1177 / 1050651916636373$

KNORR-CETINA, Karin (1981). The manufacture of knowledge. An essay on the constructivist and cContextual nature of science. Oxford: Pergamon Press.

KULGEMEYER, Christoph y PETERS, Cord (2016). Exploring the explaining quality of physics online explanatory videos. European Journal of Physics, 37(6), 1-14. DOI: 10.1088/0143-0807/37/6/065705

LATOUR, Bruno (2009). "Tarde's idea of quantification". In M. Candea (Ed.). The social after Gabriel Tarde: Debates and assessments. London: Routledge, pp. 145-162.

LÓPEZ-CANTOS, Francisco (2017). Cultura visual y conocimiento científico. Comunicación transmedia de la ciencia en la era BigData. Barcelona: UOC. 
LOPEZ-PEREZ, Lourdes y OLVERA-LOBO, María-Dolores (2016). Comunicación pública de la ciencia a través de la Web 2.0. El caso de los centros de investigación y universidades públicas de España. El Profesional de la Información, 25(3), 441-448. DOI: 10.3145/epi.2016.may.14

LUDEWIG, Julia (2017). TED Talks as an emergent genre. CLCWeb: Comparative Literature and Culture, 19(1), 1-9. DOI: $10.7771 / 1481-4374.2946$

LYOTARD, Jean-François (1979). La condition postmoderne. Raport sur le savoir. Paris: Editions du Minuit.

MATTELART, Armand (1996). La mondialisation de la communication. Paris: Presses Universitaires de France.

McCLEAN, Stephen, McCARTAN, Kenneth, MESKIN, Sheryl, GEORGES, Beronia y HAGAN, Paul (2016). Reflections on 'YouTestTube.com': An online video-sharing platform to engage students with chemistry laboratory classes. Journal of Chemical Education, 93(11), 1863-1870. DOI: 10.1021/acs.jchemed.6b00045

MESEGUER-MARTíNEZ, Ángel, ROS-GÁLVEZ, Alejandro y ROSA-GARCÍA, Alfonso (2017). Satisfation with online teaching videos: A quantitative approach. Innovations in Education and Teaching International, 54(1), 62-67. DOI: 10.1080/14703297.2016.1143859

MIJIN, Lee (2014). Metaphor Use in TED Talks: Implications for EFL. The Sociolingüistic Journal of Korea, 22(2), 65-90. DOI: 10.14353/sjk.2014.22.2.65

MORENO-CASTRO, Carolina (2011). Periodismo y divulgación científica. Tendencias en el ámbito iberoamericano. Biblioteca Nueva / OEI-Organización de Estados Iberoamericanos.

MOROZOV, Evgeny (2013). To save everything, click here: The folly of technological solutionism. New York: Public Affairs.

NEGROPONTE, Nicholas (1984). Five Predictions. TED Ideas Worth Spreading. Recuperado de: https:// www.ted.com/talks/nicholas_negroponte_in_1984_makes_5_predictions

PFOTENHAUER, Sebastian; JASANOFF, Sheila (2017). Panacea or Diagnosis? Imaginaries of Innovation and the "MIT Model" in Three Political Cultures. Social Studies of Science, 47(6), 783-810. DOI: $10.1177 / 0306312717706110$

ROBINSON, Ken (2006). Do Schools Kill Creativity?. TED Ideas Worth Spreading. Recuperado de: https://www.ted.com/talks/ken_robinson_says_schools_kill_creativity

SCHÄFER, Mike (2009). From public understanding to public engagement: An empirical assessment of changes in science coverage". Science Communication, 30(4), 475-505. DOI: $10.1177 / 1075547008326943$

SCHARRER, Lisa, RUPIEPER, Yvonne, STADTLER, Marc y BROMME, Rainer (2016). When science becomes too easy: Science popularization inclines laypeople to underrate their dependence on Experts. Public Understanding of Science, 26 (8), 1003-1018. DOI: 10.1177/0963662516680311 
SCOTTO di CARLO, Giuseppina (2014a). The role of proximity in online popularizations: The case of TED Talks. Discourse Studies, 16(5), 591-606. DOI: 10.1177/1461445614538565

SCOTTO di CARLO, Giuseppina (2014b). Humour in popularization: Analysis of humour-related laughter in TED Talks. The European Journal of Humour Research, 1, 81-93.

SEGAR, Adrian (2011). The Tyranny of TED. Conferences That Work. Recuperado de: http://www. conferencesthatwork.com/index.php/event-design/2011/10/the-tyranny-of-ted

STRAYER, Jeremy (2014). How learning in an inverted classroom influences cooperation, innovation and task orientation. Learning Environments Research, 15(2), 171-193.

SUGIMOTO, Cassidy y THELWALL, Mike(2013). Scholars on soap boxes: Science communication and dissemination via TED videos. The Journal of the American Society for Information Science $y$ Technology, 64(4), 663-674. DOI: 10.1002/asi.22764

SULESKI, Julie, IBARAKI, Motomu (2010). Scientists are talking, but mostly to each other: A quantitative analysis of research represented in mass media. Public Understanding of Science, 19(1), 115-125. DOI: $10.1177 / 0963662508096776$

TOURÓN, Javier y SANTIAGO, Raúl (2015). El modelo Flipped Learning y el desarrollo del talento en la escuela. Revista de Educación, 368, 196-231. DOI: 10.4438/1988-592X-RE-2015-368-288

TRENCH, Brian (2007). How the internet changed science journalism. In M. Bauer y M. Bucchi (Eds.), Journalism, Science and Society: Science Communication between News and Public Relations. New York/London: Routledge, pp. 133-142.

TSAI, T. (2015). Are You TED Talk material? Comparing prosody in professors and TED speakers. Proceedings of the Annual Conference of the International Speech Communication Association, 2534-2538. Recuperado de: https://www.isca-speech.org/archive/interspeech_2015/papers/115_2534.pdf

TSOU, Andrew, THELWALL, Mike, MONGEON, Philippe y SUGIMOTO, Cassidy (2014). A community of curious souls: An analysis of commenting behavior on TED Talk videos. PLOS ONE. DOI: 10.1371/ journal.pone.0093609

WANG, Qing (2012). Coaching for learning: Exploring coaching psychology in enquiry based learning and development of learning power in secondary education. Procedia - Social and Behavioral Sciences, 69, 177-86. DOI: 10.1016/j.sbspro.2012.11.397

WELBOURNE, Dustin y GRANT, Will (2016). Science communication on YouTube: Factors that affect channel and video popularity. Public Understanding of Science, 25, 706-18. DOI: $10.1177 / 0963662515572068$

WOLK, Leonardo (2007). Coaching: el arte de soplar brasas. Buenos Aires: Gran Aldea Editores. 
ZORN, Theodore, ROPER, Juliet, WEAVER, Kay y RIGBY, Colleen (2012). Influence in science dialogue: Individual attitude changes as a result of dialogue between laypersons and scientists. Public Understanding of Science, 21(7), 848-64. DOI: 10.1177/0963662510386292 